\title{
Startle Stimuli Exert Opposite Effects on Human Cortical and Spinal Motor System Excitability in Leg Muscles
}

\author{
T. V. ILIC ${ }^{1,2}$, M. PÖTTER-NERGER ${ }^{1}$, I. HOLLER ${ }^{1}$, H. R. SIEBNER ${ }^{1}$, N. V. ILIC ${ }^{3}$, \\ G. DEUSCHL ${ }^{1}$, J. VOLKMANN ${ }^{1,4}$
}

${ }^{1}$ Clinic of Neurology, Christian-Albrechts-University of Kiel, Germany, ${ }^{2}$ Department of Clinical Neurophysiology, Military Medical Academy, Belgrade, ${ }^{3}$ Clinic of Rehabilitation Medicine, Clinical Center of Serbia, Belgrade, Serbia, ${ }^{4}$ Clinic of Neurology, Universitatklinikum Würzburg, Germany

Received November 22, 2010

Accepted March 30, 2011

On-line July 19, 2011

\begin{abstract}
Summary
Increased excitability of the spinal motor system has been observed after loud and unexpected acoustic stimuli (AS) preceding $\mathrm{H}$-reflexes. The paradigm has been proposed as an electrophysiological marker of reticulospinal tract activity in humans. The brainstem reticular formation also maintains dense anatomical interconnections with the cortical motor system. When a startling AS is delivered, prior to transcranial magnetic stimulation (TMS), the AS produces a suppression of motor evoked potential (MEP) amplitude in hand and arm muscles of healthy subjects. Here we analyzed the conditioning effect of a startling AS on MEP amplitude evoked by TMS to the primary motor leg area. Ten healthy volunteers participated in two experiments that used a conditioning-test paradigm. In the first experiment, a startling AS preceded a suprathreshold transcranial test stimulus. The interstimulus interval (ISI) varied between 20 to 160 ms. When given alone, the test stimulus evoked a MEP amplitude of approximately $0.5 \mathrm{mV}$ in the slightly preinervated soleus muscle (SOL). In the second experiment, the startling AS was used to condition the size of the H-reflex in SOL muscle. Mean MEP amplitude was calculated for each ISI. The conditioning AS suppressed MEP amplitude at ISIs of 30-80 ms. By contrast, H-reflex amplitude was augmented at ISIs of 100200 ms. In conclusions, acoustic stimulation exerts opposite and ISI-specific effects on the amplitude of MEPs and H-reflex in the SOL muscle, indicating different mechanism of auditory-to-motor interactions at cortical and spinal level of motor system.
\end{abstract}

\section{Key words}

Transcranial magnetic stimulation - Startling acoustic stimulus • H-reflex • Motor cortex

\section{Corresponding authors}

J. Volkmann, Josef-Schneider-Str. 11, 97080 Würzburg, Germany. Fax: +49-931-201-23946.

E-mail: Volkmann_J@klinik.uni-wuerzburg.de or

T.V. Ilic, Outpatient Neurology Service and Dept of Clin Neurophysiology, $4^{\text {th }}$ floor, r. 52, Military Medical Academy, Crnotravska 17, 11000 Belgrade, Serbia. Fax (office): ++381 11 3608.745. E-mail: tihoilic@gmail.com

\section{Introduction}

Sudden, unexpected and very loud acoustic stimulus (AS) can activate startle motor response, which is characterized by a generalized cranio-caudal sequence of motor activation, affecting different muscle groups, distinctive on electromyographic testing (Brown et al. 1991). This effect has been attributed mainly to subcortical activity of the neurones in the nucleus reticularis pontis caudalis of the reticular formation (Davis et al. 1982).

Previous studies on healthy humans used startling AS to condition the size of the H-reflex in leg muscles and measured the relative change of the mean H-reflex amplitude of various inter-stimulus intervals (ISI) in a conditioning-test paradigm. The H-reflex is a reflex consistently obtained after electrical stimulation of sensory fibers and considers equivalent of the stretch reflex, predominantly characterized by the monosynaptic and oligosynaptic projections of group Ia afferents onto 
homonymous motoneurons. However, in addition to extensive use in clinical neurophysiology, H-reflex can be used as an unambiguous measure of the excitability of the motoneuron pool (Ilic and Deuschl 2005).

The strongest auditory $\mathrm{H}$ reflex facilitation was achieved for ISIs around 75-125 msec, suggesting activation of slow conducting descending pathways (Delwaide et al. 1995). Along with the data from lesioning experiments in animals, it was assumed that the auditory startle response is mediated through reticulo-spinal pathways (Yeomans et al. 1996). Reticular nuclei involved in the startle response maintain also dense interconnections with the cortical motor system. Recently, two independent research groups have demonstrated suppression of the contralateral motor evoked response (MEP) amplitude in hand and arm muscles, if slightly suprathreshold magnetic test stimulus was preceded by a sudden, surprise AS in humans (Furubayashi et al. 2000, Kuhn et al. 2004). This effect was not present when transcranial magnetic stimulation (TMS) was substituted by transcranial electric stimulation, implicating the cortical origin of MEP amplitude inhibition. The reciprocal action of startle stimuli on reticulospinal and corticospinal system may serve to suppress voluntary motor action in potentially dangerous situations that require fast reflexive motor responses.

Since these potentially complex effects of acoustic stimulation at different levels of the motor system have not been explored for the leg muscles, we analyzed the conditioning effect of a startling AS on the MEP amplitude evoked by TMS to the primary motor leg area and compared the effect to startle conditioned $\mathrm{H}$ reflexes of the soleus muscle.

\section{Methods}

\section{Subjects}

Neurophysiological examinations were performed in 10 healthy volunteers (mean age $33.18 \pm 3.63$ years, range 29-39; 2 women). All subjects were righthanded as tested with the Edinburgh inventory (Oldfield 1971). Written informed consent was obtained from all subjects. The study was performed according to the declaration of Helsinki and was approved by the ethics committee of Christian-Albrechts University of Kiel, Germany.

\section{EMG responses}

Subjects were seated comfortably in a reclining chair. Surface EMG was recorded from the soleus (SOL) and tibialis anterior (TA) muscle of the right leg, using surface electrodes in belly-tendon montage. After amplification with filters set at $10 \mathrm{~Hz}-2 \mathrm{kHz}$ the EMG signal was passed through a CED micro 1401 laboratory interface (Cambridge Electronic Design, Cambridge, UK) and fed into a personal computer (sampling rate $5 \mathrm{kHz}$ ), using customized data collection and conditional averaging software (Signal for Windows, ver. 2.13, Cambridge Electronic Design, Cambridge, UK) for offline analysis.

\section{Transcranial magnetic stimulation}

TMS was applied over the vertex through a figure-of-eight coil (outer diameter of each loop, $90 \mathrm{~mm}$; peak magnetic field $\sim 1.5 \mathrm{~T}$ ) using Mag Pro X100 magnetic stimulator (Medtronic, Skovlunde, Denmark) throughout all measurements. The stimulating coil was placed flat on the skull in medio-lateral orientation with the handle pointing to the right. Thus, the current induced in the brain was directed approximately perpendicular towards the assumed line of the interhemispheric sulcus (biphasic reversal mode). This is the optimal orientation for a predominantly trans-synaptic activation of the corticospinal neurone (Di Lazzaro et al. 2001, Kaneko et al. 1996). The optimal coil position for activating the right SOL was determined as the site where stimulation produced consistently the largest MEP at a slightly suprathreshold stimulus intensity. This site was marked with a pen in order to assure a constant placement of the coil throughout the experiment which was fixed on the holder. TMS intensity was adjusted to produce a target response amplitude of $0.5 \mathrm{mV}(0.3-0.7 \mathrm{mV})$, peak-to-peak in the slightly preinervated SOL muscle. During experimental sessions subjects were encouraged to maintain a slight level of contraction in the right SOL muscle.

H-reflex

In order to evoke the Soleus $\mathrm{H}$ reflex, the stimulating electrodes were placed in the upper part of the popliteal fossa over the posterior tibial nerve. The leg was fixed with the knee flexed at 120 degrees and the ankle at 100 degrees. A single rectangular shock (1 ms) was given at an intensity that induced an $\mathrm{H}$ reflex with an amplitude of $30 \%$ of the Soleus maximal motor response $(8.51 \pm 5.04 / \mathrm{SD} / \mathrm{mV})$. To record the $\mathrm{H}$ reflex EMG surface, electrodes were inserted over the Achilles tendon and $3-5 \mathrm{~cm}$ proximally. The ground was placed over the calf. The signals were amplified (gain 1000), filtered 

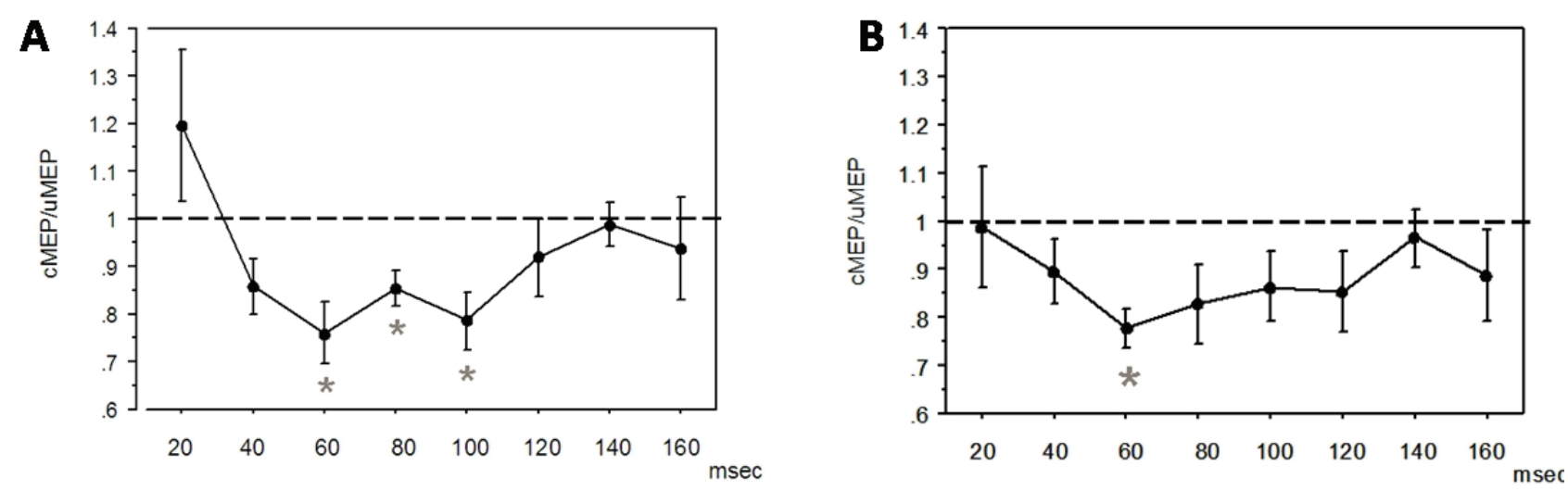

Fig. 1. Mean conditioned/unconditioned (c/u) MEP amplitude in slightly preinnervated SOL muscle (A) and resting TA muscle (B). Conditioning AS preceded TMS by 8 different ISI, which are plotted on the $x$-axis. All data are means $\pm S E(N=10)$. Significant suppression was evoked at ISIs of $60-100 \mathrm{~ms}$ in SOL muscle, and at ISI of $60 \mathrm{~ms}$ in TA muscle. The asterisk marks a significant difference $(P<0.05$; ANOVA test, post-hoc analysis).

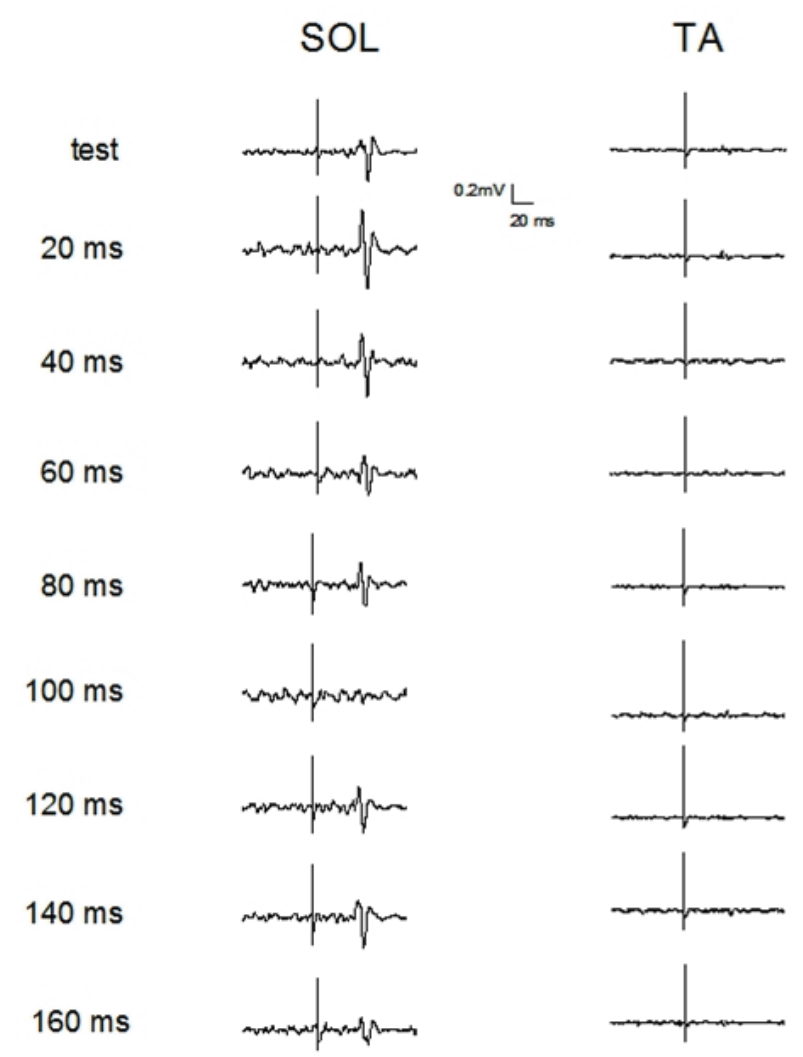

Fig. 2. Averaged data traces from one representative subject showing the effect of conditioning AS on MEP amplitudes in SOL and TA. Each trace is the average of fifteen responses, except for test response where 30 MEPs were averaged.

(bandpass filter between $80 \mathrm{~Hz}-2.5 \mathrm{kHz}$ ) and digitised (sampling frequency $5 \mathrm{kHz}$ ).

\section{Conditioning auditory stimulation}

The test H-reflex was conditioned by preceding binaural AS $(100 \mathrm{~ms}, 100 \mathrm{~dB}, 500 \mathrm{~Hz})$ delivered through headphones at 9 different randomly alternated ISI $(0,50$,

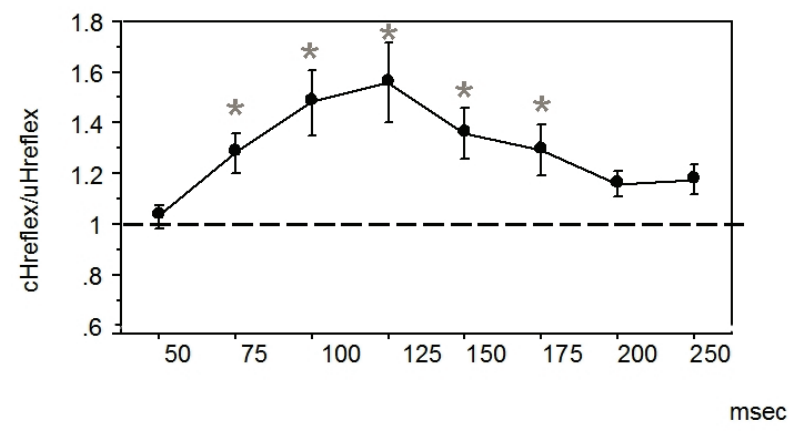

Fig. 3. Average ratio change in H-reflex amplitude for right SOL muscle. Each point is the mean $( \pm \mathrm{SE})$ of the data from ten subjects, and each value is the average of ten responses. Responses at ISIs 75-175 ms are significantly larger compared to test values $(P<0.05$; ANOVA test).

$75,100,125,150,175,200,250 \mathrm{~ms})$. Stimulation was repeated randomly at intertrial intervals (ITI) of 20-30 seconds. For each ISI, 10 H-reflexes were recorded and compared to 30 randomly intermixed unconditioned reflexes.

In the second experiment, startling AS with the same characteristics are used to condition the size of MEPs in SOL muscle. The ISIs varied between 20-160 (eight steps of $20 \mathrm{~ms}$ increments). For each ISI condition 15 MEPs were recorded and compared to 30 randomly intermixed unconditioned responses.

The amplitude change of the conditioned reflexes was expressed as a percentage of unconditioned control values.

\section{Statistical analysis}

The normalized mean values of MEPs or $\mathrm{H}$ reflexes were tested in an one-factor ANOVA model with ISI as the main effect ( 8 levels). Based on the 
significance of the F-value, the different time intervals were compared with the control responses by Bonferronicorrected multiple paired t-tests. The significance level for all tests was set to $\mathrm{p}<0.05$ (two-sided).

\section{Results}

Conditioned MEP amplitude following TMS of the cortical leg area

The test MEP amplitude in right SOL muscle could be maintained at the target level of approximately $0.5 \mathrm{mV}(0.64 \pm 0.29 \mathrm{mV})$ with a TMS intensity of $64.67 \pm 12.64 \%$ maximal stimulator output. Conditioning of the MEP amplitude by preceding sudden loud AS evoked a MEP inhibition curve (Fig. 1a with a slight to moderate conditioning effects at ISIs of 60,80 and $100 \mathrm{~ms}$ $(\mathrm{p}<0.05)$. The inhibitory effect was observed in the resting right TA muscle (MEP amplitude $0.23 \pm 0.08 \mathrm{mV}$ ), but exclusively at the ISI of $60 \mathrm{~ms}$ (Fig. 1b, p<0.05). Fig. 2. shows averaged MEPs in a single illustrative case.

In contrast to these findings, the inhibitory effect was saturated when the test MEP amplitude was adjusted to $1 \mathrm{mV}$.

\section{Conditioned H-reflex}

Conditioning the H-reflex in SOL muscle with a preceding auditory stimulation yielded a consistent pattern of responses across the various subjects. Figure 3 shows average H-reflex responses in the right SOL muscle with clear facilitation. In the intervals $75-175 \mathrm{~ms}$ marked increase in H-reflex amplitude was observed $(p<0.05)$. The maximum of the averaged conditioned response was $153.2 \%$ of the control response size at the ISI of $125 \mathrm{msec}$.

\section{Discussion}

The main finding to emerge from this study was that auditory preconditioned MEP has revealed significant amplitude decrease, in contrast to H-reflex facilitation in leg muscles under the similar circumstances.

Loud and sudden auditory stimuli elicit strong electromyographic (EMG) responses in various muscles throughout the length of the body, representing the so called startle reflex. Animal models have implicated the ventrocaudal part of the nucleus reticularis pontis caudalis of the reticular formation to generating the startle reflex. Studies using electrical stimulation of the medullary reticular formation have suggested that generalized motor inhibition can be induced via strong connections which activate spinal cord motoneurones through reticulospinal projections (Drew and Rosignol 1990, Jankowska et al. 1968, Takakusaki et al. 2001, Takakusaki et al. 1989, Takakusaki et al. 1994).

In healthy subjects, auditory stimulus at $90 \mathrm{~dB}$ induced not only SOL $\mathrm{H}$ reflex facilitation with a peak after 75-125 msec but also in the antagonistic TA muscle, and in hand muscles (Delwaide and Schepens 1989). Taking into account the latency differences between arm and leg muscles and the distance over the spine, the conduction velocity of the descending facilitatory wave was calculated to be around $20 \mathrm{~m} / \mathrm{sec}$, that corresponds to a very slow conducting descending pathway other than the corticospinal tract. The same conduction velocity was also estimated from animal experiments (Iwamoto et al. 1990, Wu et al. 1988).

In recent studies auditory preconditioning TMS has induced clear inhibitory effects, on TMS to arm (Kuhn et al. 2004) and hand muscles (Furubayashi et al. 2000. By contrast, transcranial electric stimulation (TES) in first dorsal interosseous muscle of healthy volunteers didn't change MEP amplitude at ISIs of 30-60 ms, while late facilitation (ISI of $80 \mathrm{~ms}$ ) was observed (Furubayashi et al. 2000). In line with those findings, subcortical electrical stimulation through deep electrodes implanted in subthalamic nucleus of five implanted Parkinsonians evoked facilitation (Kuhn et al. 2004). Both approaches promote evidence against a possible interaction of acoustic stimulation at the subcortical level, with motor evoked potentials. In agreement with those observations, it has been speculated that the startle brainstem system can exert opposite effects on spinal and cortical motoneurones (Valls-Sole et al. 1999). It was speculated that loud and sudden auditory stimulus activate synchronously reticulocortical and reticulospinal projections.

So far, studies of auditory preconditioned MEP have been performed exclusively in upper limb muscles. In this study we decided to combine auditory H-reflex modulation in SOL muscle in line with auditory preconditioned TMS. Spinal reflex mechanisms are preferentially evaluated in human leg muscles due to the phylogenically distinct spinal mechanisms subserving stance and walking. It might be assumed that a strong spinal level facilitation mediated via descendent reticulospinal projections at least partially cancelled an inhibition of the cortical motor pathway following 
audiogenic startle stimuli, which could explain the relatively modest modulation of auditory preconditioned MEP compared to the strong facilitation of auditory preconditioned H-reflexes.

Studies of the interaction between cortical and spinal brainstem motor systems in patients with various neurological diseases (e.g. gait improvement in Parkinsonian where patients underwent deep brain stimulation) can provide better understanding of pathophysiological mechanisms.

In summary, here we show for the first time motor cortical inhibition following auditory stimulation in a leg muscle, indicating similar mechanisms also confined to the motor cortex area representing hand and arm muscles.

\section{Conflict of Interest}

There is no conflict of interest.

\section{Acknowledgements}

TVI was a fellow of European Federation of Neurological Societies (Department-to-department Cooperation Programme).

\section{References}

BROWN P, ROTHWELL JC, THOMPSON PD, BRITTON TC, DAY BL MARSDEN CD: The hyperekplexias and their relationship to the normal startle reflex. Brain 114: 1903-1928, 1991.

DAVIS M, GENDELMAN DS, TISCHLER MD, GENDELMAN PM: A primary acoustic startle circuit: lesion and stimulation studies. J Neurosci 2: 791-805, 1982.

DELWAIDE PJ, SCHEPENS B: Auditory startle (audio-spinal) reaction in normal man: EMG responses and H reflex changes in antagonistic lower limb muscles. Electroencephalogr Clin Neurophysiol 97: 416-423, 1995.

Di LAZZARO V, OLIVIERO A, SATURNO E, PILATO F, INSOLA A, MAZZONE P, PROFICE P, TONALI P, ROTHWELL JC: The effect on corticospinal volleys of reversing the direction of current induced in the motor cortex by transcranial magnetic stimulation. Exp Brain Res 138: 268-273, 2001.

DREW T, ROSSIGNOL S: Functional organization within the medullary reticular formation of intact unanesthetized cat. II. Electromyographic activity evoked by microstimulation. J Neurophysiol 64: 782-795, 1990.

FURUBAYASHI T, UGAWA Y, TERAO Y, HANAJIMA R, SAKAI K, MACHII K, MOCHIZUKI H, SHIIO Y, UESUGI H, ENOMOTO H, KANAZAWA I: The human hand motor area is transiently suppressed by an unexpected auditory stimulus. Clin Neurophysiol 111: 178-183, 2000.

ILIC TV, DEUSCHL G: Spinal and long-latency reflexes. In: Clinical Neurophysiology of Motor Neuron Disease. Handbook of Clinical Neurophysiology, Volume 4. EISEN A (ed), Elsevier Ltd, 2004, pp 337-348.

IWAMOTO Y, SASAKI S: Monosynaptic excitatory connections of reticulospinal neurones in the nucleus reticularis pontis caudalis with dorsal neck motoneurones in the cat. Exp Brain Res 80: 277-289, 1990.

JANKOWSKA E, LUND S, LUNDBERG A, POMPEIANO O: Inhibitory effects evoked through ventral reticulospinal pathways. Arch Ital Biol 106: 124-140, 1968.

KANEKO K, KAWAI S, FUCHIGAMI Y, SHIRAISHI G, ITO T: Effect of stimulus intensity and voluntary contraction on corticospinal potentials following transcranial magnetic stimulation. J Neurol Sci 139: 131-136, 1996.

KUHN AA, SHAROTT A, TROTTENBERG T, KUPSCH A, BROWN P: Motor cortex inhibition induced by acoustic stimulation. Exp Brain Res 158: 120-124, 2004.

OLDFIELD RC: The assessment and analysis of handedness: the Edinburgh inventory. Neuropsychologia 9: 97-113, 1971.

TAKAKUSAKI K, KOHYAMA J, MATSUYAMA K, MORI S: Medullary reticulospinal tract mediating the generalized motor inhibition in cats: parallel inhibitory mechanisms acting on motoneurons and on interneuronal transmission in reflex pathways. Neuroscience 103: 511-527, 2001.

TAKAKUSAKI K, OHTA Y, MORI S: Single medullary reticulospinal neurons exert postsynaptic inhibitory effects via inhibitory interneurons upon alpha-motoneurons innervating cat hindlimb muscles. Exp Brain Res 74: 1123, 1989. 
TAKAKUSAKI K, SHIMODA N, MATSUYAMA K, MORI S: Discharge properties of medullary reticulospinal neurons during postural changes induced by intrapontine injections of carbachol, atropine and serotonin, and their functional linkages to hindlimb motoneurons in cats. Exp Brain Res 99: 361-374, 1994.

VALLS-SOLE J, ROTHWELL JC, GOULART F, COSSU G, MUNOZ E: Patterned ballistic movements triggered by a startle in healthy humans. J Physiol 516: 931-938, 1999.

WU MF, SUZUKI SS, SIEGEL JM: Anatomical distribution and response patterns of reticular neurons active in relation to acoustic startle. Brain Res 457: 399-406, 1988.

YEOMANS JS, FRANKLAND PW: The acoustic startle reflex: neurones and connections. Brain Res Rev 21: 301-324, 1996. 